\title{
Doubts hang
}

\section{over source of bird flu spread}

The H5NI avian flu virus has spread to Africa and the European Union for the first time. Attention is focused on controlling the outbreaks, especially in Nigeria, where, as Nature went to press, four farms in two adjacent provinces were confirmed as being affected, with further spread suspected. But experts are also urgently trying to confirm the cause of the virus's geographical spread. Although migratory birds have been widely blamed, some believe that the risks posed by the poultry trade are being overlooked.

Until last summer, the virus was restricted to hotspots in a handful of countries in southeast Asia. But since then it has fanned out across the globe, crossing Eurasia into Europe and Africa. The disease's appearance in Africa marks a huge leap in its range, and opens a new front that could vastly increase the bird reservoir that could spark a human pandemic (see Nature 437, 1212-1213; 2005). As in southeast Asia, rural communities often depend heavily on poultry farming, and live in close contact with both domestic and migratory birds.

The outbreak began in Jaji village, in Kaduna state in northern Nigeria, on 10 January and killed 40,000 battery chickens, although it was reported to the World Organization for Animal Health only on 6 February.

Over the weekend, outbreaks in wild swans were reported for the first time in Greece, Italy, Bulgaria and Slovenia. Swans are often sentinels of the pres-

\section{"Continued focus on} wild birds is misguided and is channelling away valuable resources." published this month also found that H5NI was present in otherwise healthy migratory birds in overwintering sites in southern China just before their migration (H. Chen et al. Proc. Natl Acad. Sci. USA 103, 2845-2850;2006).

But it is difficult to be sure whether migratory birds are sparking new outbreaks in poultry, or whether they pick up the virus from poultry infected by other routes. The same study, which analysed more than 13,000 virus samples, also found that trade and poultry movements were by far the biggest factor driving both the repeated spread of the virus outwards from its cradle in ence of wider infections, as their large size means their deaths are the first to be noticed.

Most public-health efforts are focused on preventing further spread of the virus in Africa, and urging local populations to avoid butchering and other practices that could lead to human cases. But carrying out an effective assessment of which species pose the highest risk and which areas could be hit next, as well as setting up appropriate monitoring and control measures, depends on knowing how thevirus is spreading. And on this issue experts are divided.

Many blame migrating birds. The gene sequence of the virus in Nigeria is identical to that found in Turkey last summer, and the country is a major flyway for several migratory birds from Eurasia. One large genetic study southern China and its genetic diversity. "The link [to migratory birds] seems to remain circumstantial and speculative," says Nick Davidson, deputy secretary-general of the Swiss-based Ramsar Convention on Wetlands.

"No clear evidence is available," agrees Salim Javed, an ornithologist at the Environmental Research and Wildlife Development Agency in Abu Dhabi. He adds that if migratory birds had played a major role he would have expected widespread outbreaks across India, the Middle East and Africa during the autumn migration. This did not happen.

Outbreaks may have occurred in other African countries but not been reported, says Juan Lubroth, a senior official at the United Nations' Food and Agriculture Organization.
“There may be points in between that we don't know about, that might connect the dots," he says. Indeed, international agencies are tracking rumours of outbreaks in several African countries, including Mali, Egypt, Malawi and Libya. But Lubroth agrees that "it is too easy to point at wild birds". In many African countries a there is scant surveillance of travellers carrying eggs and other poultry products, he points out.

Richard Thomas, a spokesman for Birdlife International, says he is "increasingly concerned that the continued focus on wild birds as the principal means of transmission of the virus is both misguided and a dangerous channelling away of valuable resources". Countries with strict bans on poultry imports from neighbouring infected countries, such as Myanmar, South Korea and Malaysia, are currently free of the disease.

There are previous cases of smuggling and the legal poultry trade causing long-distance spread of H5N1, adds Thomas. He says a 2004 poultry outbreakin Lhasa, Tibet, was traced to the transport of birds from Lanzhou in Chinas Gansu province, 1,500 kilometres away. ${ }^{\text {"T }}$ The death of poultry from an identical viral strain in locations many kilometres apart says nothing about how the virus was transferred between the two places." Major railwaylines linksouthern China to Kazakhstan, Russia and Eastern Europe, and many believe these could be a route for transmission of the virus from Asia to Eurasia. Declan Butler 